\title{
The Quality Evaluation of Tea (Camellia sinensis) Varieties Based on the Metabolomics
}

\author{
Zhongshuai Gai ${ }^{1}$ \\ College of Life Science, Yantai University, Yantai, Shandong 264005, China
}

Yu Wang ${ }^{1}$, Jutang Jiang, Hui Xie, and Zhaotang Ding ${ }^{2}$

Tea Research Institute, Qingdao Agricultural University, Qingdao 266109, China

Shibo Ding and Hui Wang
Rizhao Tea Research Institute of Shandong, Rizhao, Shandong 276800,
China

Additional index words. germplasm resources, metabolomics, tea [Camellia sinensis (L.) O. Kuntze]

\begin{abstract}
The identification and evaluation of tea [Camellia sinensis (L.) O. Kuntze] germplasm resources are of great significance for tea plant breeding. In recent years, various methods, such as morphology, biochemistry, molecular markers, and sensory evaluation, have been used to evaluate the tea germplasm resources. However, the evaluation of tea germplasms based on metabolomics is rarely reported. In this study, we first measured the main agronomic characters and biochemical components of tea young shoots in spring, and then analyzed the metabolic profiles using gas chromatographymass spectrometry (GC-MS) and liquid chromatography with tandem mass spectrometry (LC-MS/MS). The results indicate that tea germplasm accessions QN3 and QN38 had excellent agronomic traits with early germination and high yield compared with $H M$. The biosynthesis of flavonoids in young shoots of QN3 was more vigorous, especially for the biosynthesis of epigallocatechin gallate (EGCG) and epicatechin gallate (ECG). Accession QN3 had highest content of luteoloside, myricetin and rutin, whereas QN38 had highest content of most amino acids. On the basis of sensory quality evaluation, accession QN3 and QN38 all had higher total quality scores. By using these approaches, we found that QN3 and QN38 are excellent breeding materials with high yield and high quality for making green teas. We also believe that the evaluation system constructed by the approaches described here is suitable for the identification of tea germplasms.
\end{abstract}

Tea [Camellia sinensis (L.) O. Kuntze] germplasms are valuable fundamental materials for tea breeding and biotechnology research. Large variations in the phenotypic and biochemical characters of tea germplasms presented abundant resources for expanding the breeding program (Das and Bhagobaty, 2010).

Received for publication 31 Oct. 2018. Accepted for publication 12 Dec. 2018.

This work was subsidized by the technology system of modern agricultural industry in Shandong Province (SDAIT-19-01) and Special Foundation for Distinguished Taishan Scholar of Shandong Province (No.ts201712057). We thank BioNovoGene for technical assistance.

Zhongshuai Gai carried out the experiment, collected and organized data, and wrote the manuscript. Yu Wang and Jutang Jiang participated in designing the experiment and directed the study. Zhaotang Ding, the corresponding author, raised the hypothesis underlying this work, designed the experiment, and helped organize the manuscript structure. Shibo Ding and Hui Wang, helped conduct the experiment and collect and organize data.

${ }^{1}$ These authors contributed equally to this work. ${ }^{2}$ Corresponding author. E-mail: dzttea@163.com.
Therefore, the germplasm appraisal using different biochemical parameters affecting tea quality is essential for selecting desirable varieties. In recent years, many germplasm appraisal methods, such as morphology, biochemistry, molecular markers, and sensory evaluation, have been used to evaluate tea germplasm resources (Feng et al., 2014; Li et al., 2016; Wambulwa et al., 2016). Phenotype can be referred to a good standard for the evaluation of tea germplasm. The simplest method for genetic diversity assessment is based on the morphological traits (Gunasekare, 2007). For example, the tea yield depends on the leaf size, shoot density, bud weight, and germination period, whereas tea quality mainly depends on the biochemical components in tea shoots. Research on these biochemical components can help gain an understanding of how they affect the quality characteristics of tea varieties (Chen and Zhou, 2005; Kilel et al., 2013). More recently, the technology of molecular markers has proven to be one of the most effective methods for identifying different tea varieties (Chen et al., 2005; Chen and Yamaguchi, 2010; Yao et al., 2008).

Metabolomics has become a powerful platform that can be used to investigate genetic diversity at the metabolic level (Fernie and Klee, 2011; Langridge and Fleury, 2011). Metabolomics approach has also been used to investigate the relationship between the biochemical components and the growth conditions of tea plants (Lee et al., 2013, 2015). However, using the metabolomics approach to evaluate tea germplasm has rarely been reported.

For several years, we found that two tea germplasm accessions, QN3 and QN38, grown in the tea germplasm resource nursery at Qingdao Agricultural University, had good agronomic characteristics. To further identify and evaluate the quality of these two tea germplasms for tea breeding, we measured the biochemical components of young shoots in spring and analyzed the metabolite profiles using GC-MS and LC-MS/MS. This study not only established an evaluation system for tea germplasm appraisal but also promoted germplasm utilization for tea breeding.

\section{Materials and Methods}

Plant materials. The tea germplasm accessions QN3, QN38, and HM (used as reference), all aged 5 years, were planted in the tea germplasm resource nursery of Rizhao Station at the Tea Research Institute of Qingdao Agricultural University in Shandong province of China $\left(35^{\circ} 04 \mathrm{~N}, 119^{\circ} 39 \mathrm{E}, 53.68 \mathrm{~m}\right.$ above sea level). The young shoots with two leaves and a bud of each cultivar were plucked randomly from 10 plants on 25 May 2017. These samples were subsequently divided into two parts: one part was immediately frozen in liquid nitrogen and stored at $-80{ }^{\circ} \mathrm{C}$ until freeze-drying. The lyophilized samples were used for metabolome analysis with six biological replications. The other part was dried at $80{ }^{\circ} \mathrm{C}$ for physiological determinations. In addition, the shoots of the three varieties were also harvested to produce green teas.

Measurement of morphological variations. The main morphological characters of these tea varieties were documented, including sprouting time, sprout densities (number of shoots $/ 0.1 \mathrm{~m}^{2}$ ), leaf shape (ratio of leaf length to leaf width), leaf size (leaf length $\times$ leaf width $\times 0.7, \mathrm{~cm}^{2}$ ), and weight of 100 shoots $(\mathrm{g})$.

Determinations of tea quality components. The contents of caffeine, water extract, and free amino acids were measured in accordance with a previous report (Chen and Zhou, 2005).

Total tea polyphenols were extracted and determined spectrophotometrically according to the standard method established by the International Organization for Standardization $14502-1$ as described by Han et al. (2016). Gallic acid was used as standard. In brief, the diluted sample extract $(1.0 \mathrm{~mL})$ was transferred to tubes in duplicate, where each tube contained $5.0 \mathrm{~mL}$ of a $1 / 10$ dilution of Folin-Ciocalteu's reagent in water. Afterward, $4.0 \mathrm{~mL}$ sodium carbonate solution $(7.5 \% \mathrm{w} / \mathrm{v})$ was added into each tube. The tubes were kept at room temperature for 
$60 \mathrm{~min}$ before absorbance at $765 \mathrm{~nm}$ was measured against water.

Catechins were extracted from leaves as described previously (Fan et al., 2015). The extracts of standards and samples were subjected to high-performance liquid chromatography (HPLC) analysis using a C18 column (Agilent Technologies Inc., Santa Clara, CA). The mobile phase A was double distilled water/acetonitrile/acetic acid/EDTA $(88.8 / 9 / 2 / 0.2 ; \mathrm{v} / \mathrm{v} / \mathrm{v})$, The mobile phase B was double distilled water/acetonitrile/acetic acid/EDTA (17.8/80/2/0.2; v/v/v). The samples were eluted at a flow rate of $1 \mathrm{~mL} / \mathrm{min}$. The eluate was analyzed by absorbance at $278 \mathrm{~nm}$. The identities of each catechin were determined by comparing each retention time to the retention times of authentic standards. The HPLC-grade standards included EC, EGC, C, ECG, and EGCG, and were purchased from Sigma (St. Louis, MO). Three independent extractions were performed for each sample.

Determination of the chlorophyll content. The chlorophyll content was measured in accordance with previous report (Lichtenthaler, 1987). Data were analyzed by analysis of variance with Duncan's multiple range tests.

Identification of metabolites using $G C$ $M S$. The frozen shoots $(100 \mathrm{mg})$ were grinded into fine powders under liquid nitrogen by a chilled pestle and mortar. Metabolite extraction for GC-MS was carried out as described in our previous research (Shen et al., 2015).

We introduced the quality control (QC) samples to ensure the stability of the system. To avoid system error, all the samples were injected into apparatus randomly.

Agilent 7890A/5975C GC-MS system (Agilent Technologies) was used for GC-MS and operated as our previous research (Shen et al., 2015). A $1-\mu \mathrm{L}$ derivatized sample was analyzed and identified significant substances with a 20:1 split injection ratio. The system was equipped with a HP-5 MS capillary column $(5 \%$ phenyl methyl silox: $30 \mathrm{~m} \times$ $250 \mu \mathrm{m}$ inside diameter, $0.25 \mu \mathrm{m}$; Agilent J\&W Scientific, Folsom, CA). Injection temperature was $280^{\circ} \mathrm{C}$, interface $150^{\circ} \mathrm{C}$ and the ion source got turned to $250^{\circ} \mathrm{C}$. The temperature of transfer line was kept at $285^{\circ} \mathrm{C}$. Separations were acquired according to the following temperature procedure: isothermal heating at $40{ }^{\circ} \mathrm{C}$ for $5 \mathrm{~min}$, followed by a $10^{\circ} \mathrm{C} \cdot \mathrm{min}^{-1}$ oven ramp to $300^{\circ} \mathrm{C}$, and a $5 \mathrm{~min}$ isothermal heating at $300{ }^{\circ} \mathrm{C}$. The carrier gas (helium) was maintained at a constant linear velocity at $1 \mathrm{~mL} \cdot \mathrm{min}^{-1}$.

Mass spectra were obtained by full scan monitoring mode with the mass scan range of 35 to $750 \mathrm{~m} / \mathrm{z}$ at the scan speed of $1000 \mathrm{u} / \mathrm{s}$. The ionization mode was electron impact at $70 \mathrm{eV}$, and a detector voltage was $0.9 \mathrm{kV}$.

Peaks with the signal to noise ratio $>6$ were picked up by Agilent ChemStation. Raw MS files were first turned into NetCDF format by the Agilent MSD Station and sequentially processed by XCMS software running under $\mathrm{R}$ (version 3.0.3) for chromatographic matching, metabolic features detection, and aligning all metabolite peaks of the GC-MS data.

The automated mass spectral deconvolution and identification software (AMDIS) [National Institute of Standards and Technology (NIST), Golm Metabolome Database (GMD) gmd.mpimp-mpg.de] was used to construct library and identify metabolite with a similarity of more than $80 \%$ in the raw metabolite profiles. All peak areas were then normalized.

Multivariate statistical analysis including principal component analysis (PCA) and partial least squares-discriminant analysis (PLS-DA) were carried out using Soft Independent Modeling of Class Analogy-P (version 11.0, Umetrics AB, Umea, Sweden). All variables were ultraviolet (Unit Variance) scaled before PCA and PLS-DA. Loading plot was used to show the contributions of different variables to the samples.

The loading plot in PCA and PLS-DA screened the notable metabolite differences. Subsequently, independent $t$ test was used to exclude the variables that were not significantly different $(P>0.05)$ among the groups (Excel 2007).

Hierarchical clustering analysis was carried out using $\mathrm{R}$ software (version 3.0.3) to visualize and group metabolite profiles. The data were normalized on the basis of the abundance of the internal standard and transformed with unit variance scaling.

According to pathway analysis on Metaboanalyst and Kyoto Encyclopedia of Genes and Genomes (KEGG) metabolic database (http://www.kegg.jp/), metabolic pathway was constructed.

Quantitative analysis of metabolite by $L C$ $M S / M S$. An AB SCIEX API 4000 QTRAP Mass Spectrometer was used to detect flavones in the lyophilized samples. Each sample of $100 \mathrm{mg}$ was extracted in $5 \mathrm{~mL}$ methanol. This was followed by vortex mixing for $30 \mathrm{~s}$ and transferred it to ultrasonic cleaner $\left(25^{\circ} \mathrm{C}\right)$ for $60 \mathrm{~min}$. The mixture was then centrifuged at $25^{\circ} \mathrm{C}$ and $4000 \mathrm{rpm}$ for $3 \mathrm{~min}$. The supernatant was transferred to a new plastic Eppendorf tube and dried by a vacuum concentrator. Finally, the residue was redissolved by $0.2 \mathrm{~mL}$ methanol.

Acquity Ultra Performance LC system (BEH C18 column, 2.1× $100 \mathrm{~mm}, 1.7 \mu \mathrm{m}$, Waters, Milford, MA) was used in this study and operated as described in previous research with slight modification (Degu et al., 2014) and maintained at $40{ }^{\circ} \mathrm{C}$. A sample volume of $5 \mu \mathrm{L}$ with the partial loop injection mode was used in all experiments. Autosampler temperature was maintained at $4{ }^{\circ} \mathrm{C}$. The mobile phase consisted of $0.1 \%$ aqueous formic acid (A) and methanol (B) with flow $0.25 \mathrm{~mL} \cdot \mathrm{min}^{-1}$. The following linear gradient elution was carried out: at $0-1 \mathrm{~min}$, eluent $\mathrm{B}$ $10 \% ; 1-3 \mathrm{~min}$, eluent $\mathrm{B} 10 \%$ to $33 \% ; 3-10$ min, eluent B 33\%; 10-15 min, eluent B 33$50 \% ; 15-20 \mathrm{~min}$, eluent B $50 \%$ to $90 \% ; 20$ $21 \mathrm{~min}$, eluent B $90 \% ; 21-22 \mathrm{~min}$, eluent B $90 \%$ to $10 \%$; and $22-25$ min, eluent B $10 \%$.

The mass spectrometer was equipped with an electrospray ionization (ESI) source. In the ESI, parameter settings used for the measurement were as follows: capillary voltage in negative ESI $(-4500 \mathrm{~V})$, Collision Gas (6 psi), curtain gas (30 psi), and source temperature $\left(500^{\circ} \mathrm{C}\right)$. Nitrogen was used as desolvation and cone gas. Multiple reaction monitoring was used for quantitation.

Sensory evaluation of tea samples. Sensory evaluation was carried out for tea samples according to the State Standard of China (GB/T 23776-2009) and other recommendations in the literature (Jing et al., 2017; Liu et al., 2016). Three grams of each manufactured tea sample were infused with $150 \mathrm{~mL}$ of freshly boiled water for $4 \mathrm{~min}$. Sensory evaluation of these teas and their infusions was carried out by a team of three trained panelists who assigned scores for the shape of dry tea $(20 \%)$, liquor color $(10 \%)$, aroma $(30 \%)$, flavor $(30 \%)$, and infused leaves $(10 \%)$ between 1 and 100 , with 1 being bad or "extremely disliked" and 100 being good or "extremely liked." Finally, the total scores of organoleptic quality were calculated on the basis of the weight values.

\section{Results and Discussions}

Agronomic characteristics of germplasm resources. In the present study, the three tea plants were shrub, and the tree postures were all half-expanding types (Supplemental Fig. 1A-C). The leaf shapes of QN3 and QN38 were long oval, and HM was ellipse. The leaf sizes of HM and QN3 were lobule, and QN38 was middle-large. The sprout densities of QN3 and QN38 were significantly higher than HM $(P<0.05)$ (Supplemental Fig. 1D), and sprouting times were earlier than HM in the spring (Supplemental Fig. 1E). The weight of 100 shoots of HM, QN3, and QN38 was, on average, $38 \mathrm{~g}, 42 \mathrm{~g}$, and $55 \mathrm{~g}$, respectively. The fruit shapes of the three varieties were different. The fruit shape of HM was kidney-shaped, with two seeds, whereas the fruit shape of QN3 was spherical, with only one seed. The fruit shape of QN38 was triangular, with three seeds. On the whole, QN3 and QN38 had earlier germination and higher yield compared with HM.

Biochemical components in different germplasms. As shown in Table 1, the content of total chlorophylls was highest in HM and lowest in QN3, whereas there were no obvious differences in the content of carotenoid and the ratio of Chla/b among the three varieties. The content of total catechins in QN3 was highest and lowest in QN38. The contents of tea polyphenols in HM, QN3, and QN38 were 224.44, 265.0, and 205.35 g. $\mathrm{kg}^{-1}$, respectively. This indicated that QN3 had relatively higher content of tea polyphenols. The content of amino acids in HM was highest $\left(40.85 \mathrm{~g} \cdot \mathrm{kg}^{-1}\right)$, but there was no difference between QN3 and QN38, although the content of caffeine was lowest in QN38 $\left(14.71 \mathrm{~g} \cdot \mathrm{kg}^{-1}\right)$. In addition, the ratio of polyphenols to amino acids in tea leaves is an important evaluation index for suitability of making teas (Ai et al., 2011). It is suitable for making green teas when the ratio of 
Table 1. The contents of biochemical compositions in the shoots of the three varieties.

\begin{tabular}{|c|c|c|c|}
\hline Compound $\left(\mathrm{g} \cdot \mathrm{kg}^{-1}\right)$ & HM & QN3 & QN38 \\
\hline Chlorophyll A & $1.95 \pm 0.05 \mathrm{a}$ & $1.47 \pm 0.05 \mathrm{c}$ & $1.66 \pm 0.02 b$ \\
\hline Chlorophyll B & $0.86 \pm 0.03 \mathrm{a}$ & $0.56 \pm 0.05 \mathrm{c}$ & $0.71 \pm 0.02 \mathrm{~b}$ \\
\hline Total Chlorophyll & $2.81 \pm 0.07 \mathrm{a}$ & $2.02 \pm 0.08 \mathrm{c}$ & $2.37 \pm 0.02 b$ \\
\hline Carotenoid & $0.36 \pm 0.01 \mathrm{a}$ & $0.32 \pm 0.03 \mathrm{a}$ & $0.30 \pm 0.01 \mathrm{a}$ \\
\hline Chl A/Chl B & $2.26 \pm 0.03 \mathrm{a}$ & $2.69 \pm 0.26 \mathrm{a}$ & $2.34 \pm 0.06 \mathrm{a}$ \\
\hline $\mathrm{C}$ & $6.42 \pm 0.51 \mathrm{a}$ & $5.68 \pm 0.49 \mathrm{a}$ & $5.45 \pm 0.32 \mathrm{a}$ \\
\hline $\mathrm{EC}$ & $8.42 \pm 0.24 \mathrm{a}$ & $7.9 \pm 0.54 \mathrm{ab}$ & $6.56 \pm 017 b$ \\
\hline EGC & $63.89 \pm 0.22 \mathrm{a}$ & $65.63 \pm 0.25 \mathrm{a}$ & $49.43 \pm 0.96 \mathrm{~b}$ \\
\hline ECG & $27.20 \pm 1.03 \mathrm{ab}$ & $30.20 \pm 1.47 \mathrm{a}$ & $24.73 \pm 0.38 \mathrm{~b}$ \\
\hline EGCG & $89.16 \pm 3.07 \mathrm{~b}$ & $108.5 \pm 1.68 \mathrm{a}$ & $80.69 \pm 1.32 b$ \\
\hline Total catechin content & $195.09 \pm 4.58 b$ & $217.91 \pm 3.66 \mathrm{a}$ & $166.86 \pm 1.72 \mathrm{c}$ \\
\hline Catechin quality index & $1.82 \pm 0.07 \mathrm{~b}$ & $2.11 \pm 0.03 \mathrm{ab}$ & $2.13 \pm 0.06 \mathrm{a}$ \\
\hline Water extraction & $453.35 \pm 10.75 \mathrm{a}$ & $423.33 \pm 4.19 \mathrm{a}$ & $425.31 \pm 3.17 \mathrm{a}$ \\
\hline Caffeine & $18.32+0.48 \mathrm{a}$ & $18.33+0.46 a$ & $14.71+0.23 b$ \\
\hline Amino acids & $40.85 \pm 1.58 \mathrm{a}$ & $31.92 \pm 0.18 b$ & $33.83 \pm 0.51 b$ \\
\hline Tea polyphenols & $224.44 \pm 7.57 b$ & $265 \pm 6.58 \mathrm{a}$ & $205.35 \pm 2.55 b$ \\
\hline Ratio of polyphenols to amino acids & $5.53 \pm 0.39 b$ & $8.30 \pm 0.16 \mathrm{a}$ & $6.07 \pm 0.08 \mathrm{~b}$ \\
\hline
\end{tabular}

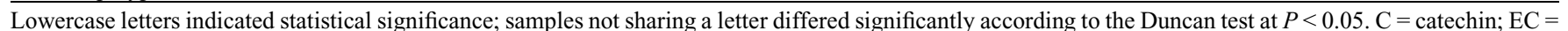
epicatechin; EGC = epigallocatechin; ECG = epicatechin gallate; EGCG = epigallocatechin gallate.

polyphenols to amino acids is less than 8 , whereas it is suitable for making black teas when it is more than 15 . In the present study, the ratio of polyphenols to amino acids in QN38 and QN3 was 6.07 and 8.30, respectively, but the ratio of polyphenols to amino acids of QN3 was closer to 8, suggesting that they all had potential values for cultivating green tea varieties.

Metabolomic analysis of young shoots. To visualize the general clustering patterns between the three varieties, we applied an unsupervised PCA approach and a supervised PLS-DA approach (Supplemental Fig. 2). As shown in the models, the three varieties were clear separation and demonstrated different metabolite profiles. Good quality parameters $(\mathrm{R} 2 \mathrm{X}=0.761, \mathrm{R} 2 \mathrm{Y}=0.982, \mathrm{Q} 2=0.756)$ from the PLS-DA model were observed, thus demonstrating that the PLS-DA model was reliable.

In total, 186 peaks were captured by GCMS, and 71 metabolites were identified by the NIST and GMD library. We chose the different metabolites by the fold changes $(\mathrm{FC} \geq 1.5$ or $\mathrm{FC} \leq 0.667)$ with significant difference $(P \leq$ 0.05 ) among the three varieties. A total of 54 metabolites were selected (Supplemental Table 1), then organized and visualized by hierarchical clustering analysis (Fig. 1). As shown in Fig. 1, the various metabolites were grouped into four clusters: A, B, C, and D. Cluster A contained 24 metabolites, such as pyroglutamic acid, valine, isoleucine, and leucine, with the highest levels in the shoots of QN38. Cluster B consisted of 12 metabolites, such as kaempferol, lysine, and proline, which were higher in QN38 and $\mathrm{HM}$ than in QN3. Cluster $\mathrm{C}$ contained 12 metabolites, such as fructose, sucrose, and maltose, which were higher in QN3 and HM than in QN38. There were six metabolites in Cluster D, such as phenylalanine, salicylic acid, and pipecolic acid, which showed the highest levels in QN3. In summary, the metabolites of the three varieties showed significant differences, of which amino acids, sugars, and organic acids dominated.

The differing metabolites between varieties were also mapped to the distinct metabolic pathways with reference to the KEGG database. These metabolites were enriched in several metabolic pathways, including alanine, aspartate, and glutamate metabolism; arginine and proline metabolism; inositol phosphate metabolism; glycine, serine, and threonine metabolism; valine, leucine, and isoleucine biosynthesis and carbon fixation in photosynthetic organisms (Fig. 2).

Changes in carbohydrate and amino acid pathways. To reveal the metabolic regulation mechanism chiefly affected by the pathways of carbohydrate and amino acid biosynthesis in the three varieties, we mapped a metabolic pathway with reference to the KEGG database and other sources (Jia et al., 2016; Li et al., 2017). As shown in Fig. 3, the various metabolites were mainly related to the carbohydrate metabolism and amino acid metabolism. The contents of sugar and alcohol metabolites were significantly different among the three varieties. The downstream compounds of sucrose, such as fructose, glucose-6-phosphate, and fructose-6-phosphate, showed more accumulations in QN3, whereas the contents of myoinositol, xylose, and myo-inositol-1-phosphate showed greater accumulation in QN38.

Much research has noted that sugars not only act as an energy and carbon source for plant growth and development but also play an important role in tea quality (Liu et al., 2016; Ruan, 2014). The sweet taste of tea infusion was shown to be due mainly to various soluble sugars, such as sucrose, fructose, and glucose (Zheng et al., 2016). In the present study, the highest content of sucrose and lower content of caffeine in QN3 might be beneficial to the taste of higher quality teas.

In addition, the various metabolites were also involved in the amino acid biosynthesis pathway (Fig. 3). Amino acids play crucial roles in plant growth and development because they are the primary nitrogen-transport compounds within plants (Urquhart and Joy, 1981), and the synthesis of amino acids is regulated by sugar supply and a variety of regulatory signals (Lam et al., 1996). In general, the content of amino acids in tea shoots is the key determinant for green tea quality (Ding et al.,
2002). High levels of free amino acids are essential for good-quality green teas. For example, albino tea cultivar, a good cultivar for making green teas, has a higher level of total amino acids and lower levels of total catechins and caffeine because the biosynthetic inhibition of catechins, and caffeine might lead to a shift in the metabolic network toward amino acid biosynthesis (Du et al., 2006; Feng et al., 2014). According to the metabolomics results (Fig. 1; Supplemental Table 1), the contents of most amino acids were the highest in QN38, suggesting that nitrogen metabolism in QN38 might be beneficial to the biosynthesis and accumulation of amino acids, which potentially results in the biosynthetic inhibition of catechins and caffeine. Previous studies showed that marked upregulation of amino acid metabolism was associated with enhanced taste of tea cultivars (Ji et al., 2017). Some amino acids, such as glutamic acid, glutamine, and aspartic acid, are recognized as main contributors to the umami taste of tea infusion (Dai et al., 2017). Similarly, the highest content of many amino acids, including glutamic acid, glutamine, and aspartic acid, was seen in QN38, suggesting that it might have good flavor for making excellent green teas.

Quantitative analysis of tea polyphenols. To further analyze the contents of tea polyphenols in shoots among the three varieties, we conducted quantitative analysis by LCMS/MS. Twenty-two kinds of flavonoid metabolites were identified, of which 16 metabolites were found to be distinct among them (Table 2). The contents of dihydromyricetin, naringin, quercetin, naringenin, kaempferol, ECG, and EGCG were significantly higher in QN38 than those in HM $(P<$ $0.05)$. The contents of dihydromyricetin, rutin, quercetin, naringenin, chrysin, ECG, EGC, and EGCG were significantly higher in QN3 than those in HM $(P<0.05)$. Specifically, the rutin, EGCG, and ECG were the most abundant secondary metabolites in the shoots of the three varieties. The contents of rutin, EGCG, and ECG were highest in QN3, whereas the contents of 


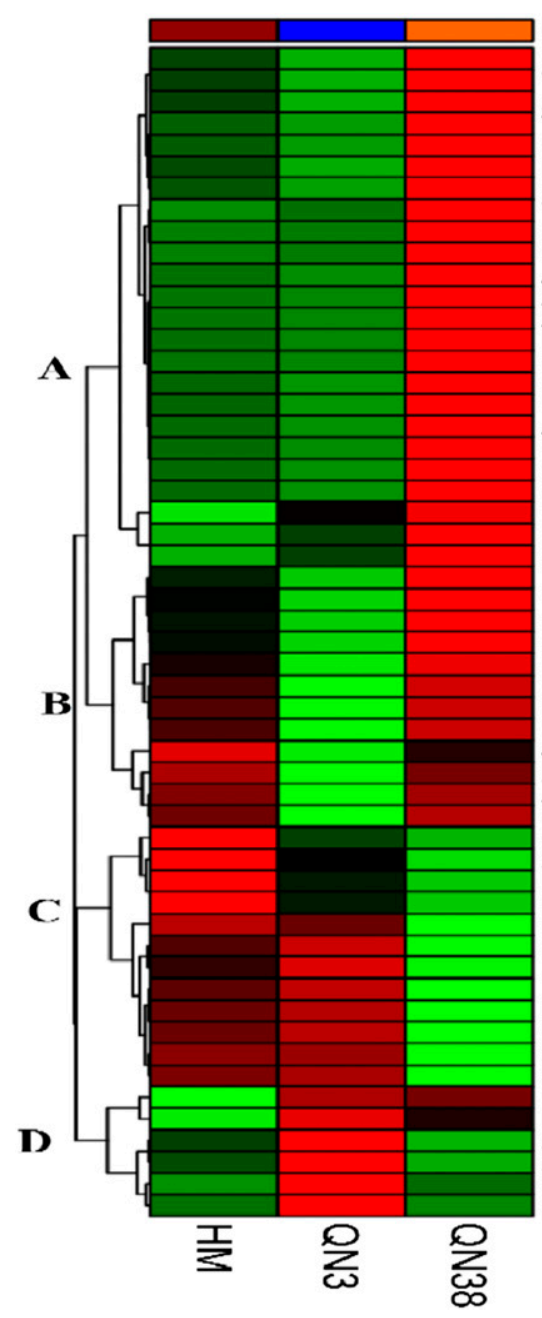

Class

Homoserine

Threonine

2,4,6-Tri-tert.-butylbenzenethiol

Valine

Glutamic acid

Pyroglutamic acid

Spermidine

Threonic acid-1,4-lactone

Rhamnose

myo-Inositol

Aspartic acid

Xylose

Arabinose

Ribonic acid

Quinic acid

Leucine

Isoleucine

Arabinitol

Glycerol-3-Phosphate

Glycine

Octadecanoic acid

myo-Inositol-1-phosphate

Phosphoric acid

Shikimic acid

Kaempferol

1-Monohexadecanoylglycerol

Malonic acid

Dehydroascorbic acid dimer

Lysine

Putrescine

1,3-Di-tert-butylbenzene

Proline

4-Aminobutyric acid

Xylitol

Alanine

Serine

Glutamine

2-Keto-L-gluconic acid

Fructose

Hexonic acid

Oxalic acid

Sorbitol-6-phosphate

Maltose

Mannose-6-phosphate

Fructose-6-phosphate

Glucose-6-phosphate

2-Ketoglutaric acid

Sucrose

Pipecolic acid

Phenylalanine

2,3-Dihydroxybutanedioic acid

2,4-5-Trihydroxypentanoic acid

Salicylic acid

Erythronic acid

Fig. 1. Hierarchical clustering of 54 metabolites identified. In this heat map, columns and rows represent samples and individual metabolites, respectively. Green indicates relatively low and red indicates relatively high intensity
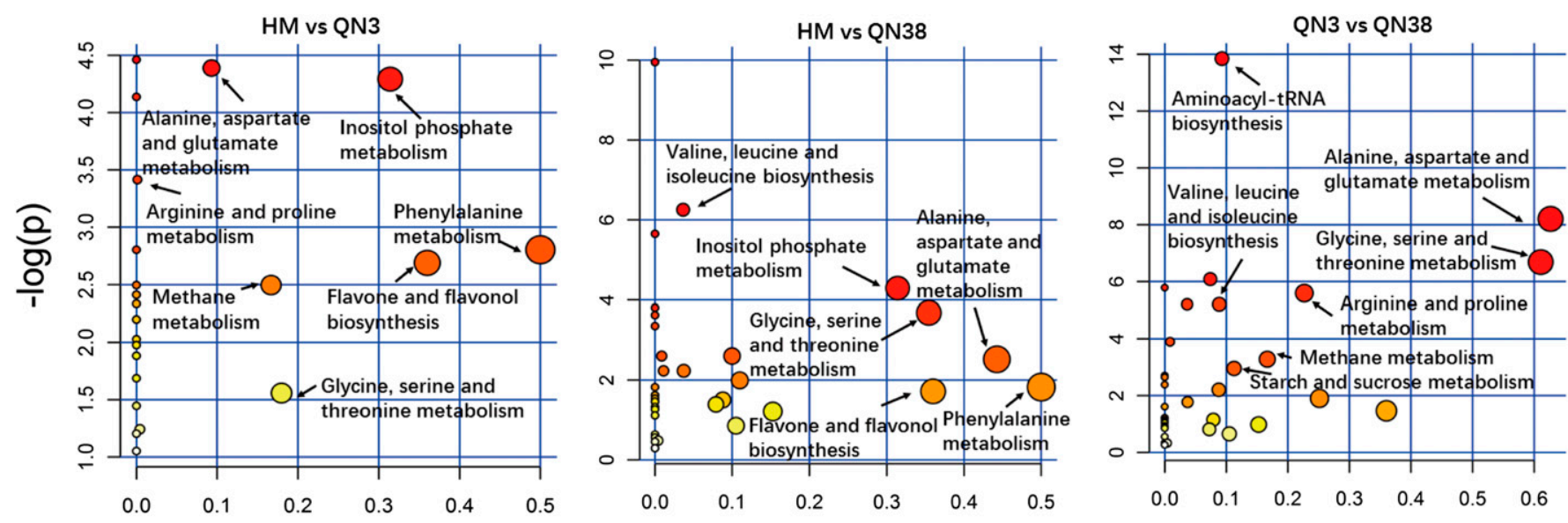

\section{Pathway Impact}

Fig. 2. Enrichment of the differential metabolites to distinct Kyoto Encyclopedia of Genes and Genomes pathways. Differential metabolites between varieties $(P \leq 0.05)$ were mapped to the distinct metabolic pathways.

EGCG and ECG were lowest in HM. The content of rutin was lowest in QN38.

Tea polyphenols, including naringin, quercetin, naringenin, kaempferol, ECG, and EGCG, were responsible for the bitterness, astringency, and sweet aftertaste of tea beverages ( $\mathrm{Li}$ et al., 2016). The level of tea polyphenols in shoots may be a reliable parameter for identifying high quality (Obanda et al., 2015). Catechins, consisting of C, EC, EGC, ECG, and EGCG, played a crucial role in tea quality. They accounted for $25 \%$ to $30 \%$ of the dry weight of tea leaves (Fan et al., 2015). The total content of catechins could be used as a biomarker for evaluating the tea quality (Magoma et al., 2000). Almost all of the 


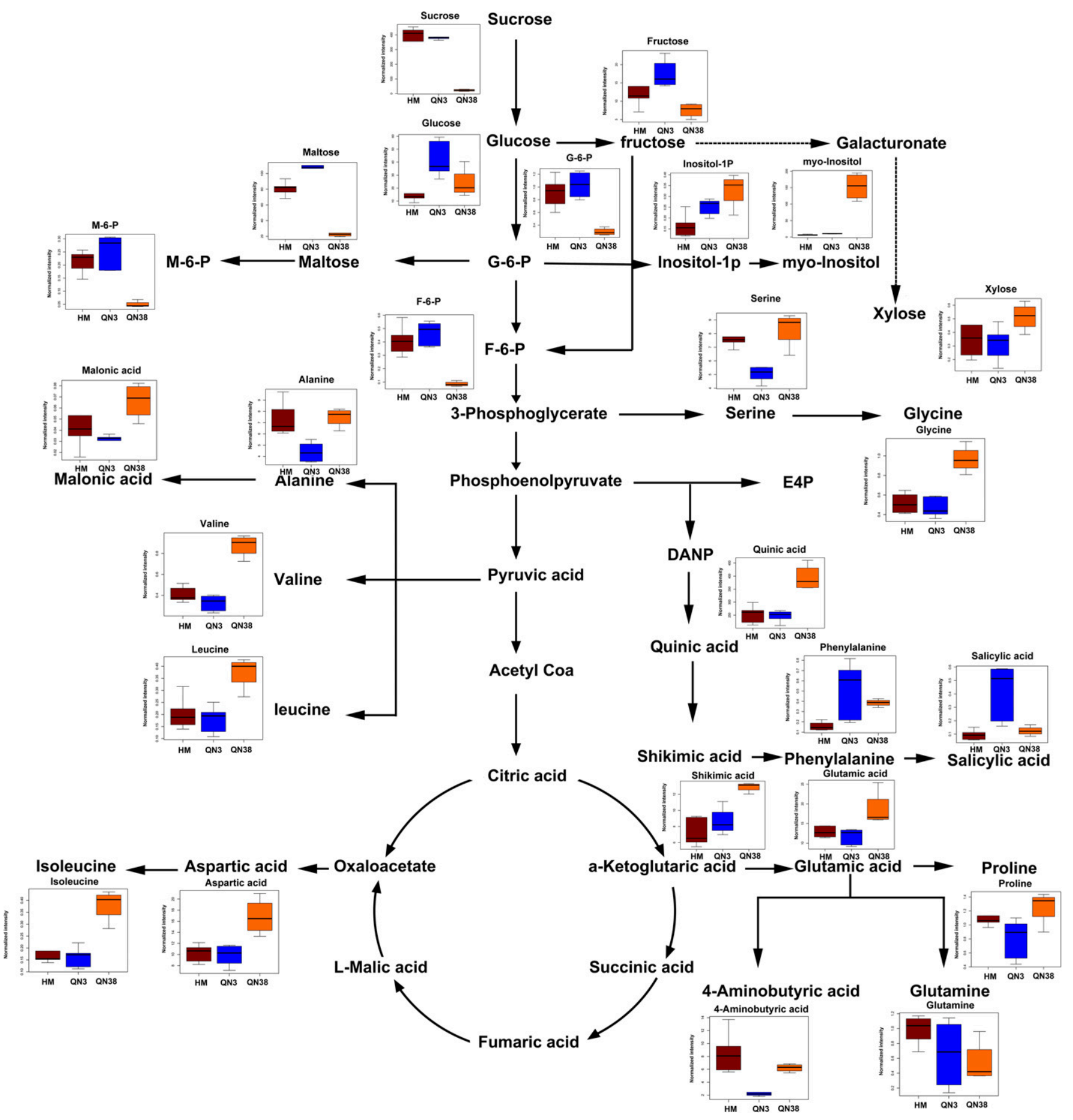

Fig. 3. Metabolomic pathway of the three varieties based on the Kyoto Encyclopedia of Genes and Genomes database. Red indicates HM, blue indicates QN3, yellow indicates QN38.

characteristics of manufactured tea, including its taste, color, and aroma, have been associated directly or indirectly with catechins (Wang et al., 2000). Our results showed that the contents of isoquercetin, luteoloside, vitexin, and myricetin, rutin, quercetin, naringenin, $\mathrm{C}, \mathrm{ECG}, \mathrm{EGC}$, and EGCG were highest in QN3, and the ratio of polyphenols to amino acids was about 8 , suggesting that QN3 might have more mellow and thick taste for making green teas.

Metabolite changes in the flavonoid biosynthesis pathway. To reveal the metabolic regulation mechanism chiefly affected the pathways of flavonoid biosynthesis in the three varieties, we mapped the flavonoid biosynthesis pathway with reference to the KEGG database. As shown in Fig. 4, we detected a strong association among naringin, apigenin, kaempferol, dihydroquercetin, and dihydromyricetin in the pathway of flavonoid biosynthesis. Dihydroquercetin and dihydromyricetin, the intermediate product of flavonoid metabolisms, plays important roles in the biosynthesis of catechins (Zhang et al., 2014). The level of dihydroquercetin was higher in QN3 than that in QN38 and HM, and the level of dihydromyricetin was higher in QN3 and QN38 than that in HM. Downstream metabolites, such as rutin, $\mathrm{C}$, and ECG deriving from dihydroquercetin, were more accumulated in QN3 than that in QN38 and HM. Similarly, EGC and EGCG derived from dihydromyricetin were more abundant in QN3 than that in HM and QN38. Interestingly, the other metabolites, such as isoquercetin, luteoloside, vitexin, isovitexin, and myricetin, were also significantly 
Table 2. The quantitative analysis of flavonoids in the shoots of the three varieties.

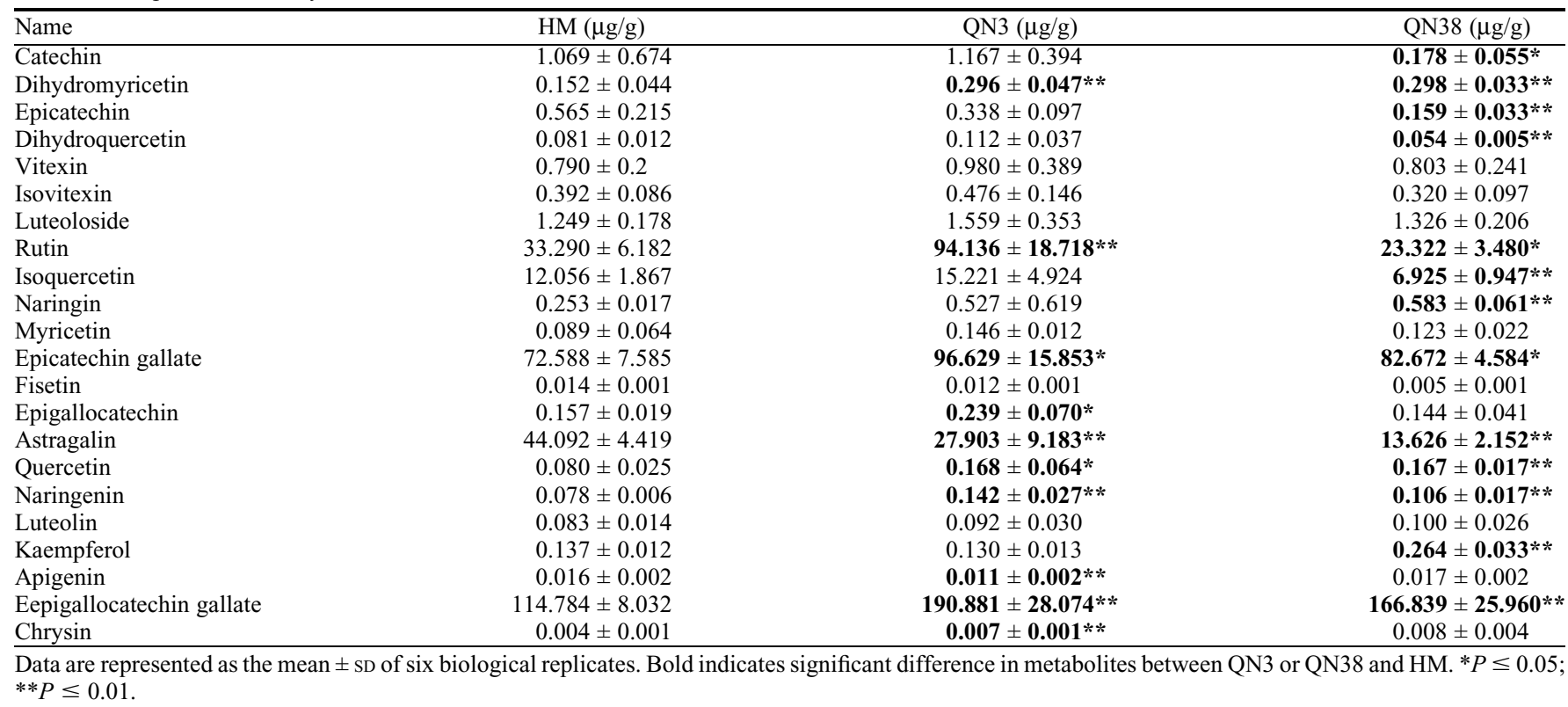

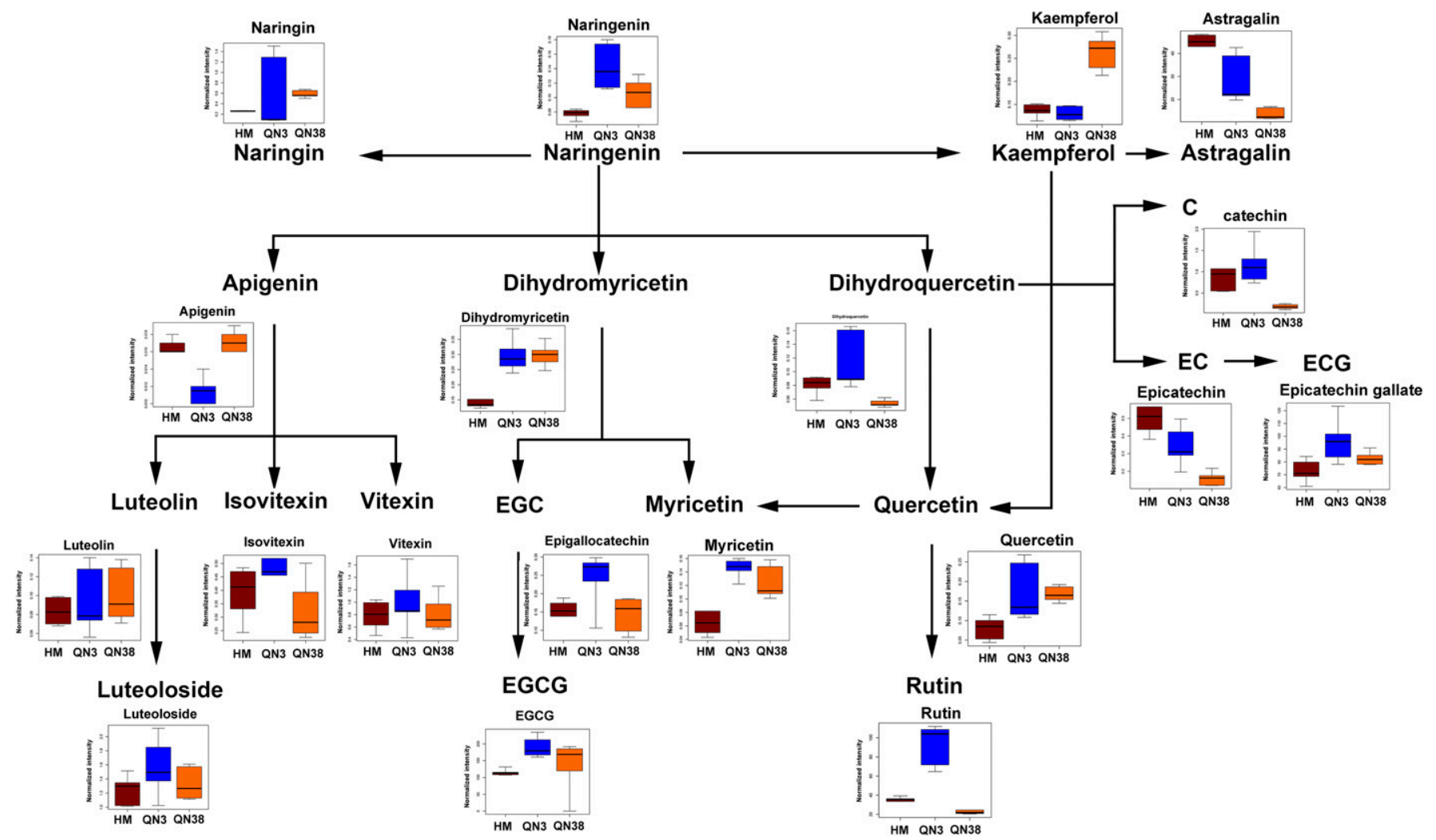

Fig. 4. Flavonoid biosynthesis pathway of three tea cultivars based on the Kyoto Encyclopedia of Genes and Genomes data set. Red indicates HM, blue indicates QN3, yellow indicates QN38.

higher in QN3 than those in QN38 and HM, indicating more vigorous biosynthesis of flavonoid metabolism in young shoots of QN3.

Sensory evaluation of three tea samples. To intuitively evaluate the qualities of these three teas, we conducted sensory quality evaluations with tea samples made from these three varieties. Total quality scores of three tea samples were all more than 90 points for the green teas (Supplemental
Fig. 3). In regard to the scores of shape, infused leaves, and liquor color, there were no differences, but there were differences in aroma and flavor. The scores of flavor and aroma of QN3 and HM were higher than those of QN38.

A delicious cup of tea infusion was believed to be a balance of various taste sensations, including sweetness, astringency, umami, and bitterness (Zheng et al., 2016). Previous studies have suggested that the relative importance of these taste sensations in green teas as follows: astringency 4.17 , bitterness 3.44, umami 1.42, and sweetness 0.53 (Sai et al., 2011). Our study showed that the contents of tea polyphenols, catechins, caffeine and soluble sugars were higher in QN3 than QN38. The results of the sensory quality evaluation were consistent with the content of the biochemical components and metabolites in the three varieties. Therefore, we speculate that the 
reason for the better flavor of QN3 in sensory evaluation might be related to the interaction of these substances, but the interaction mechanism of metabolites needs further study.

\section{Conclusion}

In the present study, we used a metabolomics approach to evaluate tea germplasms and investigate the relationship between the metabolic profiles and quality attributes of the two varieties. QN3 and QN38 were found to be excellent breeding materials with high yield and high quality for green tea. We also concluded that a metabolomics approach can be used as an effective auxiliary method to evaluate tea germplasm resources. This is of great significance for creating an evaluation system for tea germplasm resources.

\section{Literature Cited}

Ai, S.R., R.M. Wu, L.Y. Yan, and Y.H. Wu. 2011. Measurement of the ratio of tea polyphenols to amino acids in green tea infusion based on near infrared spectroscopy. Adv. Mat. Res. 301303:1093-1097.

Chen, L., Q.K. Gao, D.M. Chen, and C.J. Xu. 2005. The use of RAPD markers for detecting genetic diversity, relationship and molecular identification of Chinese elite tea genetic resources [Camellia sinensis (L.) O. Kuntze] preserved in a tea germplasm repository. Biodivers. Conserv. 14:1433-1444.

Chen, L. and S. Yamaguchi. 2010. RAPD markers for discriminating tea germplasms at the interspecific level in China. Plant Breed. 124:404-409.

Chen, L. and Z.X. Zhou. 2005. Variations of main quality components of tea genetic resources [Camellia sinensis (L.) O. Kuntze] preserved in the China National Germplasm Tea Repository. Plant Foods Hum. Nutr. 60:31-35.

Dai, W., D. Xie, M. Lu, P. Li, H. Lv, C. Yang, Q. Peng, Y. Zhu, L. Guo, and Y. Zhang. 2017. Characterization of white tea metabolome: Comparison against green and black tea by a nontargeted metabolomics approach. Food Res. Intl. 96:40-45.

Das, R.N. and R.K. Bhagobaty. 2010. Assessment of the genetic variability present in the tea germplasm of a tea garden in Assam, India using morphological and biochemical markers. J. of Tea 36:133-139.

Degu, A., U. Hochberg, N. Sikron, L. Venturini, G. Buson, R. Ghan, I. Plaschkes, A. Batushansky, V. Chalifa-Caspi, and F. Mattivi. 2014. Metabolite and transcript profiling of berry skin during fruit development elucidates differential regulation between Cabernet Sauvignon and Shiraz cultivars at branching points in the polyphenol pathway. BMC Plant Biol. 14:188.

Ding, Y., H. Yu, and S. Mou. 2002. Direct determination of free amino acids and sugars in green tea by anion-exchange chromatography with integrated pulsed amperometric detection. J. Chromatography 982:237-244.

Du, Y.Y., Y.R. Liang, H. Wang, K.R. Wang, J.L. Lu, G.H. Zhang, W.P. Lin, M. Li, and Q.Y. Fang. 2006. A study on the chemical composition of albino tea cultivars. J. Pomol. Hort. Sci. 81:809812.

Fan, K., D. Fan, Z. Ding, Y. Su, and X. Wang. 2015. Cs-miR156 is involved in the nitrogen form regulation of catechins accumulation in tea plant (Camellia sinensis L.). Plant Physiol. Biochem. 97:350-360.

Feng, L., M.J. Gao, R.Y. Hou, X.Y. Hu, L. Zhang, X.C. Wan, and S. Wei. 2014. Determination of quality constituents in the young leaves of albino tea cultivars. Food Chem. 155:98-104.

Fernie, A.R. and H.J. Klee. 2011. The use of natural genetic diversity in the understanding of metabolic organization and regulation. Front. Plant Sci. 2:59.

Gunasekare, M.T.K. 2007. Applications of molecular markers to the genetic improvement of Camellia sinensis L. (tea) - A review. J. Pomol. Hort. Sci. 82:161-169.

Han, W.Y., J.G. Huang, X. Li, Z.X. Li, G.J. Ahammed, P. Yan, and J.R. Stepp. 2016. Altitudinal effects on the quality of green tea in east China: A climate change perspective. Eur. Food Res. Technol. 243:1-8.

Ji, H.G., Y.R. Lee, M.S. Lee, K.H. Hwang, E.H. Kim, J.S. Park, and Y.S. Hong. 2017. Metabolic phenotyping of various tea (Camellia sinensis $\mathrm{L}$.) cultivars and understanding of their intrinsic metabolism. Food Chem. 233:321330.

Jia, S., W. Yu, J. Hu, Z. Ding, Q. Liang, Y. Zhang, and W. Hui. 2016. Mineral and metabolic profiles in tea leaves and flowers during flower development. Plant Physiol. Biochem. 106: 316-326.

Jing, J., Y. Shi, Q. Zhang, J. Wang, and J. Ruan. 2017. Prediction of Chinese green tea ranking by metabolite profiling using ultra-performance liquid chromatography-quadrupole time-of-flight mass spectrometry (UPLC-Q-TOF/MS). Food Chem. 221:311-316.

Kilel, E.C., A.K. Faraj, J.K. Wanyoko, F.N. Wachira, and V. Mwingirwa. 2013. Green tea from purple leaf coloured tea clones in Kenya - their quality characteristics. Food Chem. 141:769-775.

Lam, H.M., K.T. Coschigano, I.C. Oliveira, R. Melo-Oliveira, and G.M. Coruzzi. 1996. The molecular-genetics of nitrogen assimilation into amino acids in higher plants. Annu. Rev. Plant Physiol. Plant Mol. Biol. 47:569-593.

Langridge, P. and D. Fleury. 2011. Making the most of "omics" for crop breeding. Trends Biotechnol. 29:33-40.

Lee, J.E., B.J. Lee, J.O. Chung, H.N. Kim, E.H Kim, S. Jung, H. Lee, S.J. Lee, and Y.S. Hong. 2015. Metabolomic unveiling of a diverse range of green tea (Camellia sinensis) metabolites dependent on geography. Food Chem. 174:452-459.

Lee, L.S., J.H. Choi, N. Son, S.H. Kim, J.D. Park, D.J. Jang, Y. Jeong, and H.J. Kim. 2013. Metabolomic analysis of the effect of shade treatment on the nutritional and sensory qualities of green tea. J. Agr. Food Chem. 61:332338.

Li, M., R. Guo, J. Yang, X. Jin, H. Zhang, and L. Shi. 2017. Comparison of salt tolerance in soja based on metabolomics of seedling roots. Front. Plant Sci. 8:1101.

Li, Y., C. Chen, Y. Li, Z. Ding, J. Shen, Y. Wang, L. Zhao, and M. Xu. 2016. The identification and evaluation of two different color variations of tea. J. Sci. Food Agr. 96:4951-4961.

Lichtenthaler, H.K. 1987. [34] Chlorophylls and carotenoids: Pigments of photosynthetic biomembranes. Methods Enzymol. 148C:350382.

Liu, J., Q. Zhang, M. Liu, L. Ma, Y. Shi, and J. Ruan. 2016. Metabolomic analyses reveal distinct change of metabolites and quality of green tea during the short duration of a single spring season. J. Agr. Food Chem. 64:33023309.

Magoma, G.N., F.N. Wachira, M. Obanda, M. Imbuga, and S.G. Agong. 2000. The use of catechins as biochemical markers in diversity studies of tea (Camellia sinensis). Genet. Resources Crop Evol. 47:107-114.

Obanda, M., P.O. Owuor, and S.J. Taylor. 2015. Flavanol composition and caffeine content of green leaf as quality potential indicators of Kenyan black teas. J. Sci. Food Agr. 74:209215.

Ruan, Y.L. 2014. Sucrose metabolism: Gateway to diverse carbon use and sugar signaling. Annu. Rev. Plant Biol. 65:33-67.

Sai, V., P. Chaturvedula, and I. Prakash. 2011. The aroma, taste, color and bioactive constituents of tea. J. Med. Plants Res. 5:2110-2124.

Shen, J., Y. Wang, C. Chen, Z. Ding, J. Hu, C. Zheng, and Y. Li. 2015. Metabolite profiling of tea (Camellia sinensis L.) leaves in winter. Scientia Hort. 192:1-9.

Urquhart, A.A. and K.W. Joy. 1981. Use of phloem exudate technique in the study of amino acid transport in pea plants. Plant Physiol. 68:750754.

Wambulwa, M.C., M.K. Meegahakumbura, K. Samson, M. Alice, M. Michael, J. Liu, J.C. Xu, R. Sailesh, D.Z. Li, and L.M. Gao. 2016. Insights into the genetic relationships and breeding patterns of the african tea germplasm based on nSSR markers and cpDNA sequences. Front. Plant Sci. 7:1244.

Wang, H., G.J. Provan, and K. Helliwell. 2000. Tea flavonoids: Their functions, utilisation and analysis. Trends Food Sci. Technol. 11:152160.

Yao, M.Z., L. Chen, and Y.R. Liang. 2008. Genetic diversity among tea cultivars from China, Japan and Kenya revealed by ISSR markers and its implication for parental selection in tea breeding programmes. Plant Breed. 127:166172.

Zhang, Q., Y. Shi, L. Ma, X. Yi, and J. Ruan. 2014 Metabolomic analysis using ultra-performance liquid chromatography-quadrupole-time of flight mass spectrometry (UPLC-Q-TOF MS) uncovers the effects of light intensity and temperature under shading treatments on the metabolites in tea. PLoS One 9:e112572.

Zheng, C., Y. Wang, Z. Ding, and L. Zhao. 2016. Global transcriptional analysis reveals the complex relationship between tea quality, leaf senescence and the responses to cold-drought combined stress in camellia sinensis. Front. Plant Sci. 7:1858. 


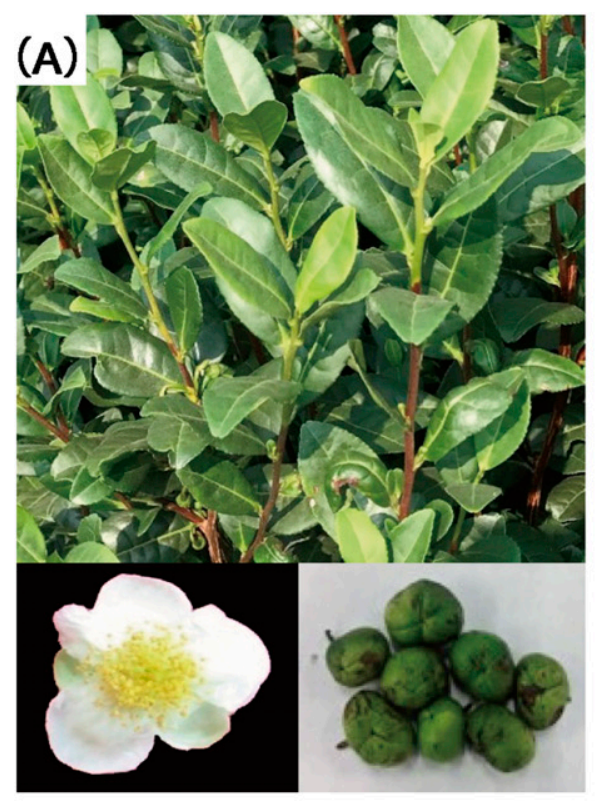

(D)

\begin{tabular}{cccc}
\hline \multirow{2}{*}{ Varieties } & \multicolumn{3}{c}{ Sprout densities (number $/ 0.1 \mathrm{~m}^{2}$ ) } \\
\cline { 2 - 4 } & April 25 & May10 & May 25 \\
\hline HM & $78.67 \pm 2.60 \mathrm{c}$ & $84.33 \pm 2.02 \mathrm{c}$ & $92.64 \pm 3.12 \mathrm{c}$ \\
QN3 & $121.33 \pm 1.76 \mathrm{a}$ & $127.33 \pm 2.60 \mathrm{a}$ & $132.29 \pm 2.90 \mathrm{a}$ \\
QN38 & $101.67 \pm 3.18 \mathrm{~b}$ & $107.6 \pm 3.84 \mathrm{~b}$ & $116.38 \pm 2.41 \mathrm{~b}$ \\
\hline
\end{tabular}
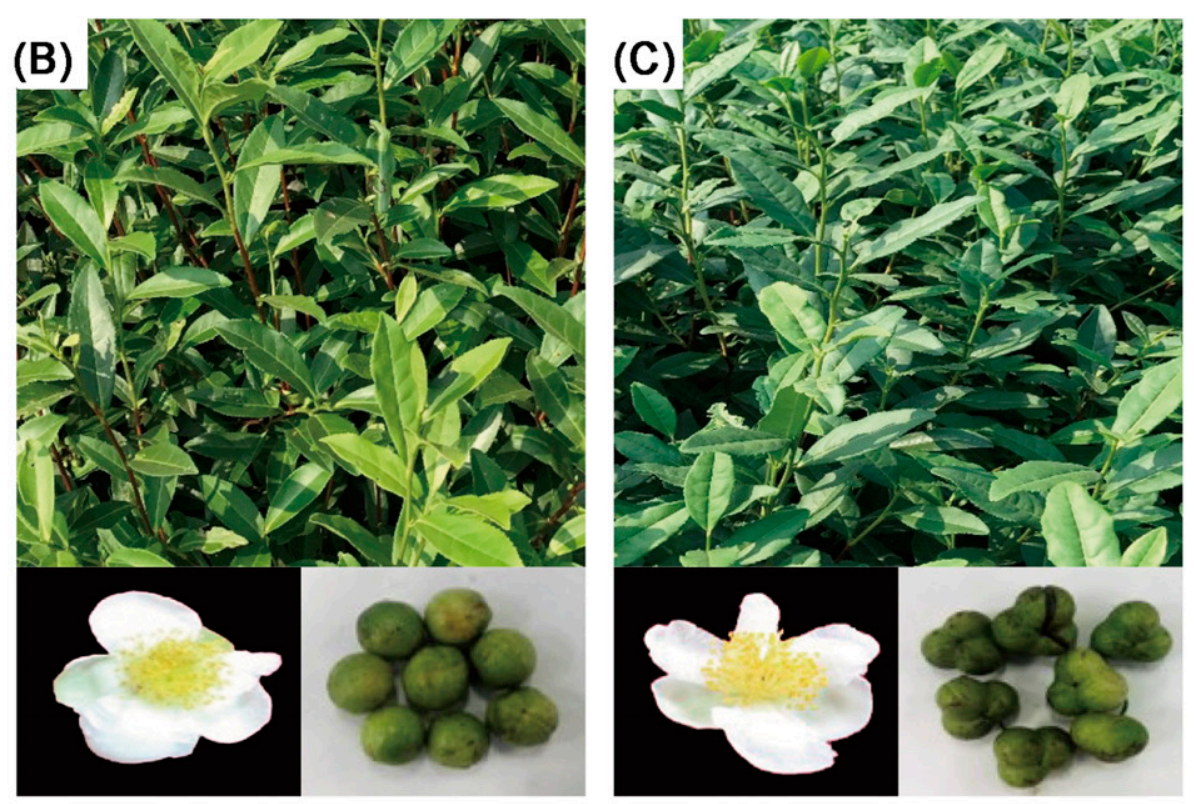

$(\mathrm{E})$

\begin{tabular}{ccccc}
\hline Varieties & $\begin{array}{c}\text { Shoots with one } \\
\text { leaves and a bud }\end{array}$ & $\begin{array}{c}\text { Shoots with two } \\
\text { leaves and a bud }\end{array}$ & $\begin{array}{c}\text { Shoots with three } \\
\text { leaves and a bud }\end{array}$ & $\begin{array}{c}\text { Period of } \\
\text { symmetry leaves }\end{array}$ \\
\hline HM & May. 5 & May. 12 & May. 19 & Jun. 6 \\
QN3 & May. 3 & May. 10 & May. 17 & Jun. 4 \\
QN38 & Apr. 22 & May. 10 & May. 16 & Jun. 6 \\
\hline
\end{tabular}

Supplemental Fig. 1. Photos and information about the three varieties: (A) HM, (B) QN3, and (C) QN38. (D) The result of sprout densities of the three varieties from 25 Apr. 2017 to 25 May 2017. (E) The germination period of the three varieties from Apr. 2017 to June 2017. Lowercase letters indicate statistical significance; samples not sharing a letter differed significantly according to the Duncan test at $P<0.05$. 
(A) PCA score plot

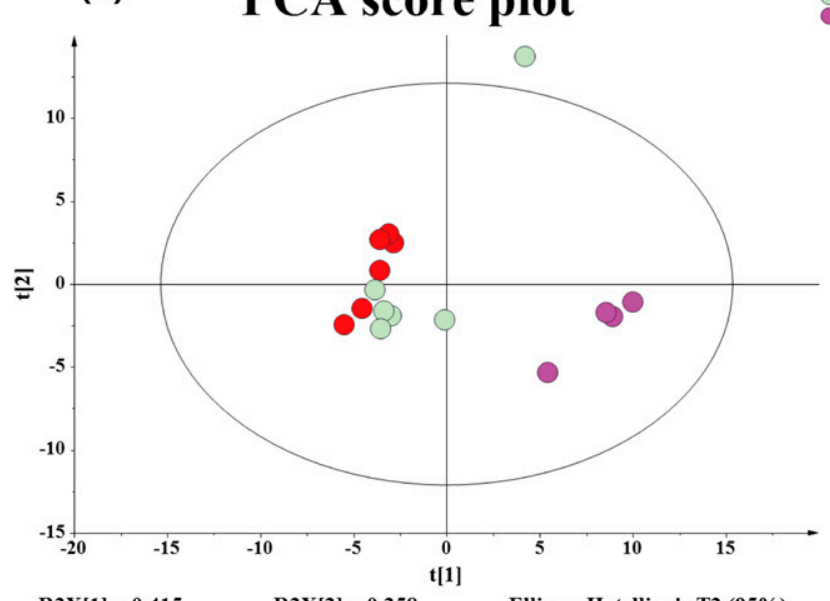

QN38

( B ) PLS-DA score plot

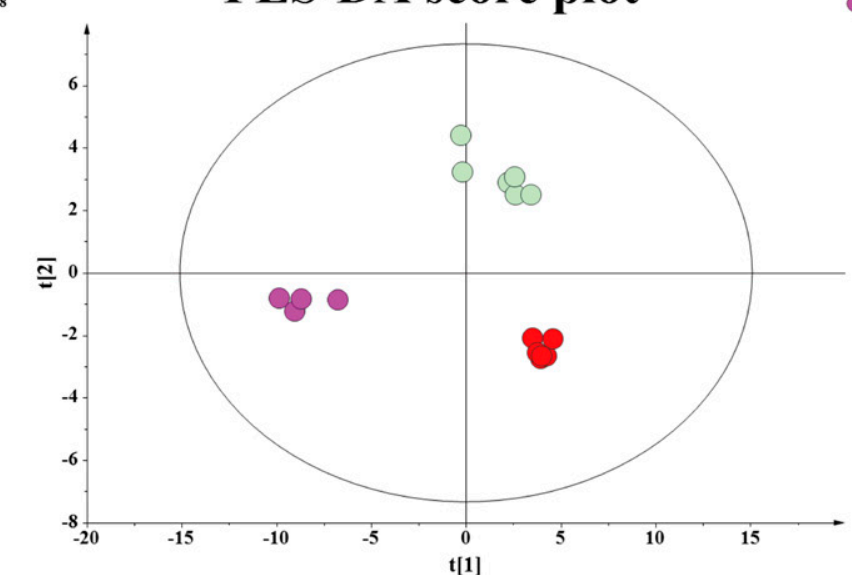

$\mathrm{R} 2 \mathrm{X}[1]=0.415$ $\mathrm{R} 2 \mathrm{X}[2]=0.258$

Ellipse: Hotelling's T2 (95\%)

$\mathrm{R} 2 \mathrm{X}[1]=0.407$

$\mathrm{R} 2 \mathrm{X}[2]=0.0987$

Ellipse: Hotelling's T2 (95\%)

Supplemental Fig. 2. Statistical and multivariate analysis of GC-MS data. (A) Principal component analysis (PCA) of metabolic profiles in the shoots of the three varieties. Quality parameters: $\mathrm{R} 2 \mathrm{X}=0.766, \mathrm{Q} 2=0.434$. (B) Partial Least Squares Discriminant Analysis (PLS-DA) of metabolic profiles in the shoots of the three varieties. Quality parameters: $\mathrm{R} 2 \mathrm{X}=0.761, \mathrm{R} 2 \mathrm{Y}=0.982, \mathrm{Q} 2=0.756$. Two abnormal samples of QN38 were removed.

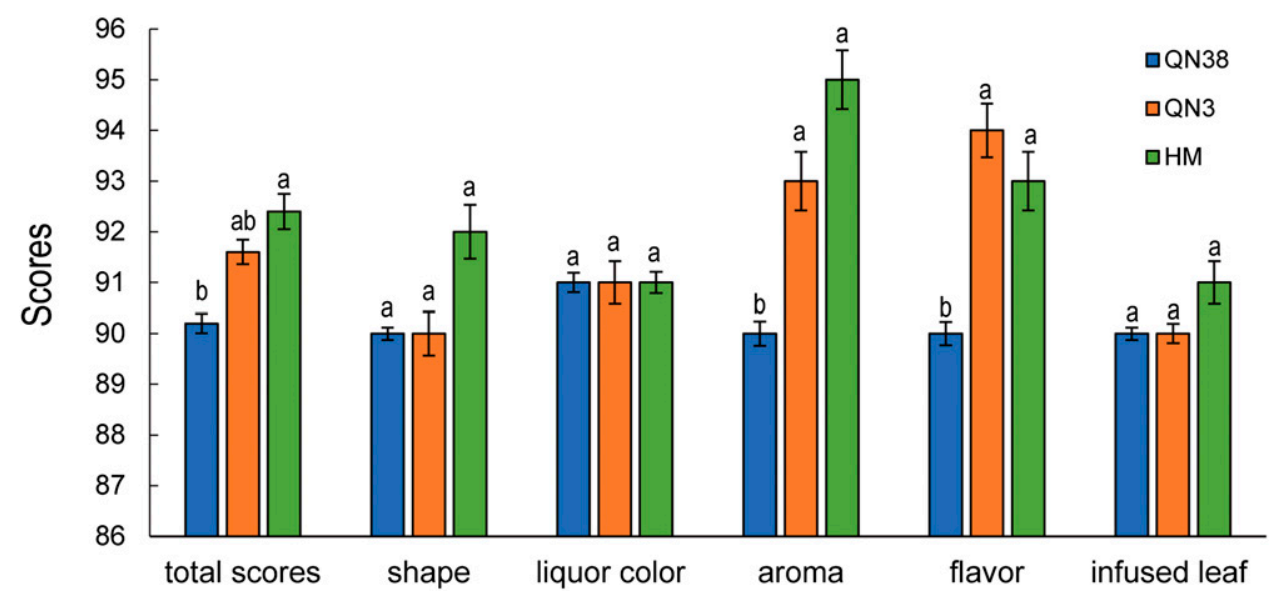

Supplemental Fig. 3. Scores for sensory evaluation of different tea variations. 
Supplemental Table 1. The fold changes of differential metabolites in the shoots of three tea varieties.

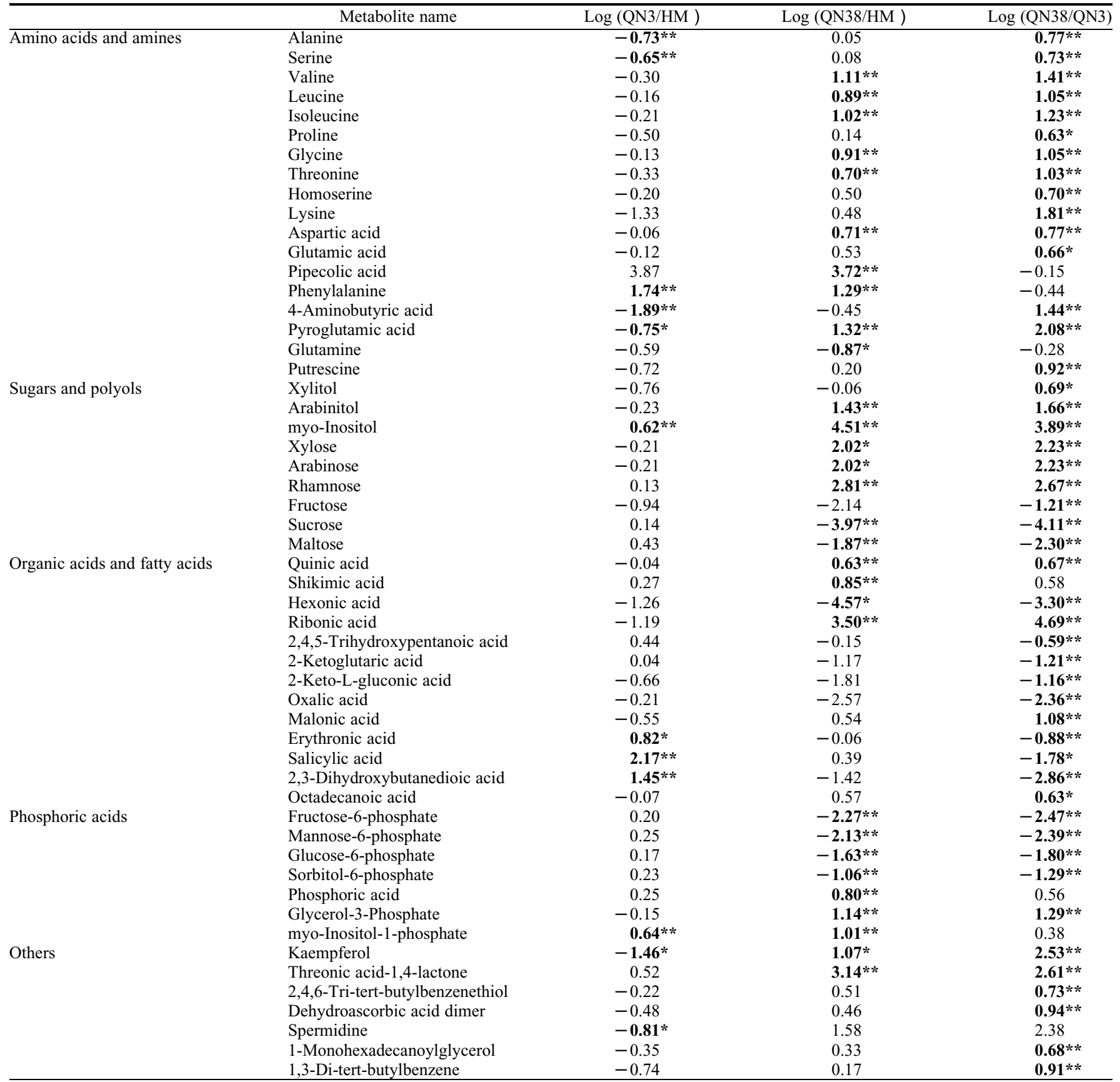

Note: The FCs were described with the $\log 2$ transformed numbers, the bold fonts indicate significant difference metabolites between varieties. The positive number indicates the increase and negative numbers indicates the decrease. ${ }^{*} P \leq 0.05 ;{ }^{* *} P \leq 0.01$. 